\title{
Nutrition by design: a review of biotechnology in functional food of plant origin
}

\section{Thomas Reynolds ${ }^{1}$ and Danik M. Martirosyan ${ }^{2}$}

${ }^{1}$ University of San Francisco, San Francisco, CA, USA; ${ }^{2}$ Functional Food Center/Functional Food Institute, Dallas, TX, USA

Corresponding author: Danik M. Martirosyan, PhD, Functional Food Center/Functional Food Institute, Dallas, TX, USA

Submission Date: December 11, 2015, Acceptance date: February 10, 2016: Publication date: February 12, 2016

\begin{abstract}
Medical institutions in industrial and developing countries are increasingly turning to functional foods as intervention in chronic disease. Advances in genetic engineering have provided methods of purposefully designing functional foods and bioactive compound-producing organisms. This literature review examines the recent history of biotechnological applications in functional food, the state of bioagricultural engineering for high-value compound production, and the challenges that developers face in promulgating functional foods from biotechnological sources. Based on the literature reviewed, it is predicted that adding biotechnologically-produced compounds will be more successful in producing novel functional foods.
\end{abstract}

Keywords: Biotechnology; functional food; genetic engineering, golden rice, algae

\section{INTRODUCTION:}

Few scientific developments of the last 50 years have had as resounding an impact on human society as biotechnology. Manipulating the nucleic acid information medium and its expression in living organisms has redefined the bounds of basic laboratory research, delivered complex macromolecular therapeutics to the clinic, and enabled material commodity production from engineered biological systems. Additionally, from a commercial perspective, the US biopharmaceutical industry's US\$110+ billion in revenue indicates that biotechnology is a lucrative proposition, as well as a transformative one [1].

While embracing the novel therapies that biotech treatment offers, medical practices and institutions are also reevaluating a very old concept: the therapeutic benefits of functional food. Precisely what constitutes a functional food depends on the definition's source, but the generally accepted definition is that a functional food, when consumed in the regular course of a person's diet, confers a well-defined health benefit beyond basal metabolic needs. The Functional Food Center's current definition [2] posits that functional foods are: 
natural or processed foods that contains known or unknown biologically-active compounds; which, in defined, effective nontoxic amounts, provide a clinically proven and strong documented health benefit for the prevention, management, or treatment of chronic disease.

This prophylactic and therapeutic potential has been drawing an increasing number of likeminded researchers, dieticians, and food producers, especially in light of chronic disease proliferation in developing and industrialized populations [2]. They also benefit from the public's positive perception and an attitudinal shift away from 'avoidance' of specific foods to 'positive eating', allowing the food industry to successfully improve the potential for 'healthy eating' [3]. The world of dietary disease treatment is growing, and the functional food concept may be a lever of sufficient length to move it.

However, the practice of functional food usage leaves much to be desired. An idealized goal for functional food, in which the consumer-patient would be able to achieve therapeutic benefit from unprocessed foods, is severely hindered by natural produce variation. A basic variable such as water availability can significantly affect the production of bioactive compound and bulk biomass, making the "defined, effective" amount of a functional food neither [4]. And even if growth characteristics could be precisely controlled for each tuber and sprig, many food items are simply not feasible bioactive compound sources in the first place. For example, quercetin is a bioactive flavonoid that has demonstrated beneficial lipidemic effects in small-scale clinical trials [5]. But these trials used concentrated Allium cepa extracts containing $100 \mathrm{mg}$ quercetin per daily dose-equivalent to $400 \mathrm{~g}$ of boiled onion, which is more than 16 times the average American's daily onion consumption as recorded in 2011 [6, 7]. Since a functional food cannot be a supplement or pill-it must be recognized as a food article and contribute to the consumerpatient's basal metabolism-many bioactive compound sources cannot provide enough bioactivity to be considered functional foods.

Therefore, many functional foods are manufactured, compounded, or otherwise processed to circumvent these limitations. A recent paper in the Journal of Cereal Science described development of a wheat pasta product functionalized with probiotic Bacillus coagulans culture, which lessened glycemic index impact in consuming subjects compared to nonfunctionalized pasta [8]. This effective functionalization hints at greater health benefits that could achieved by deliberately compounding bioactive agents in dietary staples-but why should food developers limit themselves to nature's preexisting pantry? This literature review will examine the successes and failures of food bioengineering, a few promising techniques and organisms for bioactive compound production, and the challenges still remaining in the biotechnological development of functionalized foods.

\section{TRANSGENICISM IN FOOD PRODUCTS}

A Golden Dream Deferred: As Western society entered the $21^{\text {st }}$ century, the insufficient dietary intake of vitamin A and the resulting collection of pathologies that ensued cost the eyesight of 500,000 children in developing countries and the lives of an additional 670,000 [9]. Additionally, the optimal strategy to alleviate vitamin A deficiency (VAD) is a matter of scientific debate. 
Unfortunately, the vitamin A deficiency epidemic is not dire enough to rally the scientific community together and come to a consensus over decades of arguing. However, the VAD epidemic also provides a prototypical case of functional food usage to treat chronic conditions, and a stage for the introduction of a prototypical bioengineered functional food: Golden Rice, an engineered cereal capable of endogenous $\beta$-carotene synthesis. By functionalizing this pervasive dietary staple, provitamin-A could be feasibly cultivated in the local community and provided to afflicted children [10]. Although there are many dietary natural sources of provitamin-A in the world, they frequently require industrial processing and transport (e.g. palm oil) or are otherwise too expensive for the affected population to procure (e.g. animal meat).

The genetic engineering that produced Golden Rice (GR), and its more potent successor Golden Rice 2 (GR 2), was elegantly simple-partially because very little genomic information needed to be added. The more-refined transformation element (T-DNA) used in GR 2 (see Figure 1) contains only three expressed genes: a SSUcrtI carotene desaturase-RuBisCo chloroplast transit peptide fusion, a phytoene synthase ( $p s y)$ gene from Zea mays, and a phosphomannose isomerase (pmi) gene that did not participate in pro-vitamin A synthesis, but was used for transformed rice calli selection [11]. The addition of Z. mays psy greatly improved pro-vitamin A production in GR 2, a benefit that can be visibly appreciated compared to GR transformed with psy from Narcissus pseudonarcissus (see Figure 2). Transforming the plant pathogen Agrobacterium tumefasciens with the T-DNA element, to effect gene transfer to the rice through the bacterium's infectious capacity, is a relatively blunt instrument compared to more modern genetic engineering techniques (see below). Nonetheless, A. tumefasciens is still an effective vehicle for plant transformation, and sees use today [12].

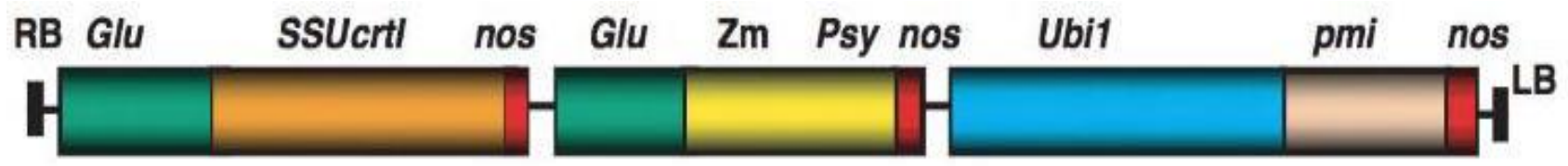

Figure 1: A schematic of the Golden Rice 2 T-DNA construct. This artificial DNA sequence combines the necessary genes for endogenous provitamin-A synthesis in Golden Rice. Glu and Ubil genes are promoters that help initiate gene expression, and nos is a terminator gene that signals the end of the gene sequence. SSUcrt, Psy, and pmi are catalytic genes described in the text. [1I]

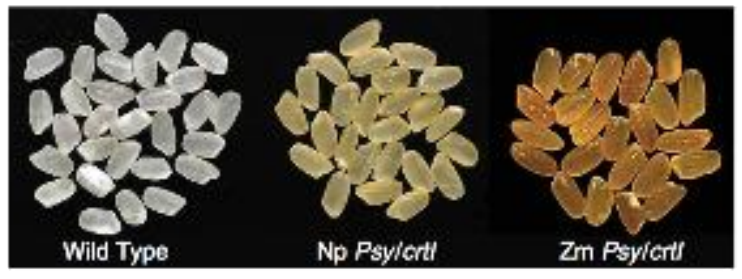

Figure 2: Visual comparison of carotenoid production in WT rice, and rice transformed with the respective $\mathrm{N}$. pseudonarcissus and $\mathrm{Z}$. mays psy transgenes. The $\mathrm{Z}$. mays psy/crtl transgenic rice's higher color saturation indicates its proportionally greater saturation in $\beta$-carotene. [11]

Unfortunately, the Golden Rice story is also an ill portent of genetic modification's sociopolitical palatability, despite its explicit benefits to human health. Global regulatory attitudes towards the production of genetically modified crops vary, from generally receptive in the United States to hostile in the European Union. Between 1992 and 2014, US regulators approved 156 applications to plant GM crops and 170 to sell GM-derived food products, while their counterparts across the Atlantic approved 67 products and 6 cultivations [13]. The greatest encumbrance on the process is perhaps that any endorsement of a transgenic crop's safety by the 
European Food Safety Authority must be approved by the European Parliament-and one in three of its members vote on an explicit platform against genetic modification of any food source [14].

Nevertheless, the European Parliament's voting record is only one legislative reflection of global society's mixed opinion on transgenic food cultivation, ranging from the supportive to the antagonistic. Fear, uncertainty, and doubt have been spread by NGOs and competing agribusiness interests, portraying the acceptance of transgenic foods as reckless and predatory, and elected policymakers have complied with this portrayal[15, 16]. Although the rice would have been made freely available to impoverished farmers, the fact that it was developed under license with a major agricorporation gave it the cursory appearance of unethically exploiting vulnerable populations. Additionally, the recent retraction and censure of Tang et al.'s study on pro-vitamin A supplementation with Golden Rice in Chinese children, possibly the single best support for its humanitarian use as a functional food, has had crippling effects on further academic interest in GM functionalization (despite the distinct taint of political motivation behind Tufts University's ethical inquest) [17]. The potential benefits of Golden Rice have been suppressed for now, by a weakness to social pathogens rather than biological.

In an important exception, regulators in Japan-the place of origin for the formal functional food concept-have apparently become much more open to genetically modified foods. Between 1992 and 2003, 58 products and 5 plantings were approved there, and has increased to 129 products and 115 plantings between 2004 and 2014 [13]. However, very few globally approved transgenic crops have been designed with the intent of improved nutritional content; producers are more interested in increased production and pest resistance. This will likely change as interest in functional food applications continues to grow.

Newer Options for Genetic Engineering: In the time since GR was developed, more refined approaches to genetic engineering have been applied to crop plants with varying degrees of success. However, the three most prominently applied methods all make use of the target organism's own DNA-repairing pathways for effecting a stable and lasting transformation. The current greatest advance in engineering, the CRISPR/Cas 9 complex, is a widely-applicable platform quickly being used in multiple domains of life. A DNA plasmid bearing the Cas 9 nuclease and a guiding RNA sequence can effect genomic edits with high specificity and efficiency, but the guide RNA is much easier to alter for different targets than the peptide-based DNA-recognition sites of previous methods (see Fig. 3) [18]. CRISPR/Cas 9 has already demonstrated its utility in a wide variety of plants, especially for multiple simultaneous gene insertions $[19,20]$. Precisely adding synthetic or accumulative genes to food items, to grow designer functional foods in the soil, is becoming a much more scientifically feasible proposition than it was even five years ago.

It also has been suggested that, if the guiding RNA and $\operatorname{Cas} 9$ nuclease are introduced directly into a cell without a DNA intermediate, a resulting organism can be considered genetically unmodified under current laws and enjoy greater public support. [21] This contention is only relevant in the EU, where transgenic regulation focuses on the processes to introduce the transformative element used, rather than on the product yielded [22]. 
The relevant regulation in the US, 7 CFR 340, only gives the Department of Agriculture power over "the introduction of organisms and products altered or produced through genetic engineering which are plant pests or which there is reason to believe are plant pests." The only

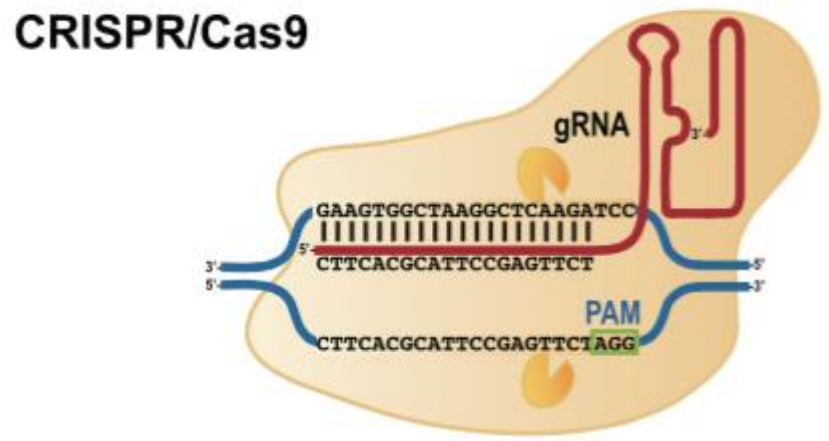

Figure 3: A simplified diagram of the CRISPR/Cas 9 complex acting on a DNA strand. The red gRNA construct directs the nuclease activity to sequences with complementarity, provided they are immediately downstream of a protospacer adjacent motif (PAM) sequence specific to Cas9 from a given bacterial species. [21]

way 7 CFR 340 would apply to CRISPR/Cas 9 engineering is if the introduced gene material comes from a plant pest, or if a plant pest such as a virus is used as the transformative agent [23]. Moreover, without an additional DNA template Kanchiswamy et al.'s ribonucleoprotein method can only silence a target gene, not augment or replace it, thereby severely reducing CRISPR/Cas9's usefulness.

Furthermore, it seems unlikely that the nuance between genetic editing and genetic modification will be appreciated, as the end result is still an organism whose genome has been altered by direct intervention. As long as elements of the public remain hostile to bioengineered agriculture, every GM crop will grow in a minefield of inflamed controversy. As a result, concluding that CRISPR/Cas 9 will usher in a new era of genetic engineering for food functionalization is entirely premature at this stage, due to its novelty and public reticence, but the prospect is an unquestionably tantalizing one.

\section{FUTURE DIRECTION: TRANSGENIC PRODUCERS, NOT PRODUCTS}

Algae for A Better Life: Embryophyta, or land plants, are by no means the only genetically engineered templates in development. Microalgal species have been favorite subjects for commodity production, realizing commercial and material value from fatty acid synthesis pathways [24]. The rich energy content of algal fatty acids, and their supposedly-sustainable production compared to fossil fuels, made biofuels the primary drivers of algal biotech development. However, the genetic engineering of microalgae to produce fuel compounds (including alcohols) represents a "fourth generation" of biofuel production methods, and still has yet to fulfill biofuel's promises of environmentally-conscious and economically competitive energy [25]. To expand the platform's utility, and provide alternative revenue streams in the meantime, engineers now offer a portfolio of microalgae-produced substances; these compounds find applications such as industrial lubricants, livestock feed, and consumer foods and cosmetics. In many circumstances compounds destined for different applications can be selectively isolated from the same culture, reducing the overall cost of production (see Fig. 4a) [24, 26]. 


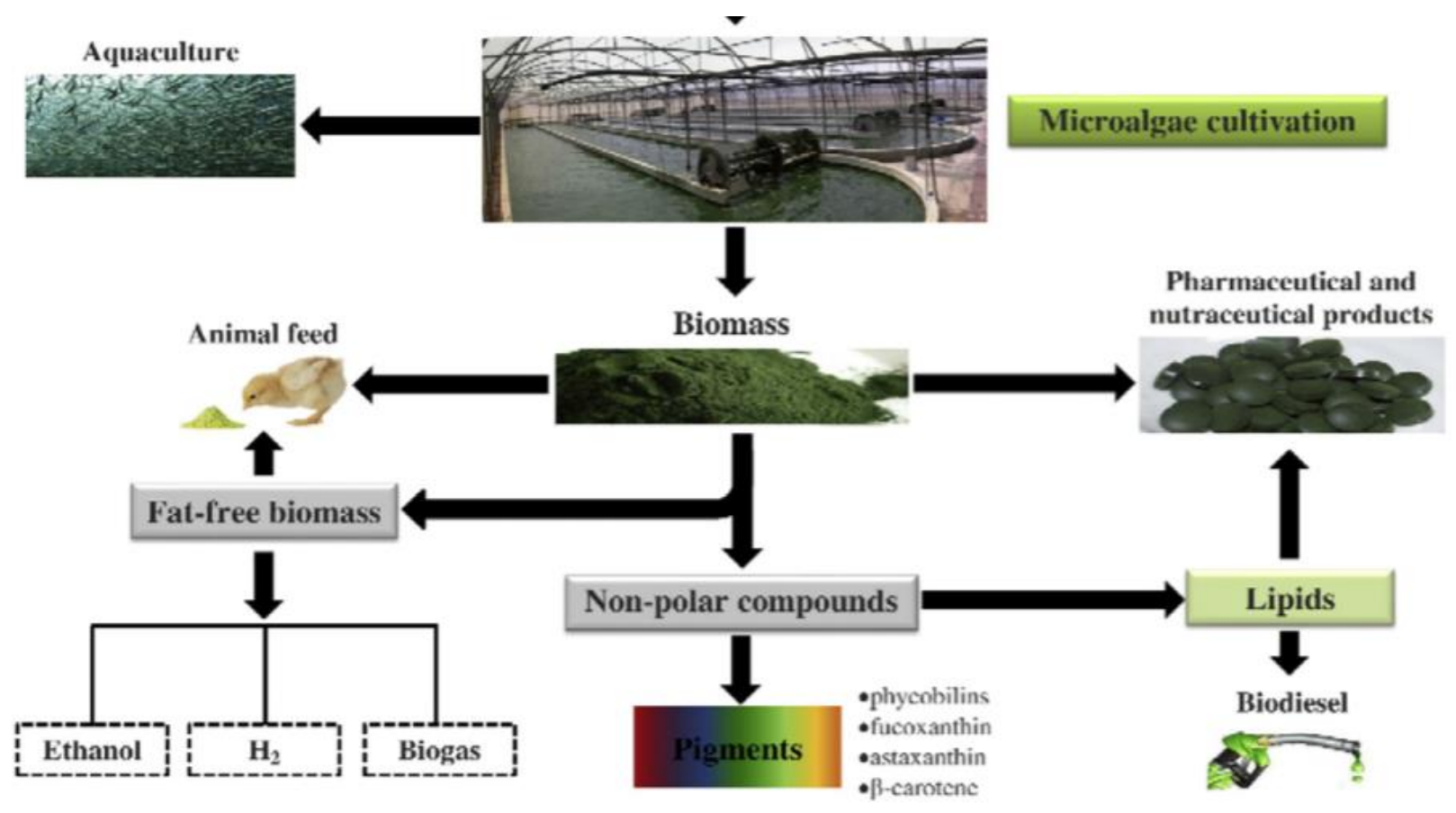

Figure 4a: A high-level conceptualization of the microalgal economy, where high-value compounds and bulk products can be produced and extracted in the same culture. The functional food community would most likely be interested in the production of biomass for pigments, lipids, and miscellaneous bioactive compounds. [23]

Polyunsaturated fatty acids (PUFAs, see Table 1), and the functional food researcher's host of bioactive compounds (see Table 2) are not only readily produced in microalgae but can also be produced in greater quantity per culture by biotechnological means [24]. Nevertheless, the entire point of algal culture for functional food production, metabolically engineered or not, is to improve consumer diet and health en mass. The cultivation of microalgae for consumption is still a niche market, despite its historical precedence, and a public already disinclined to eat microalgae is even less likely to be enticed by genetically modifying it.

\begin{tabular}{|l|l|l|}
\hline Table 1: Useful Fatty Acids [26] & Structure & Example Microalgal Source \\
\hline \}-Linolenic acid & $18: 3 \omega 6,9,12$ & Arthrospira \\
\hline Arachidonic acid & $20: 4 \omega 6,9,12,15$ & Porphyridium \\
\hline Eicosapentaenoic acid & $20: 5 \omega 3,6,9,12,15$ & $\begin{array}{l}\text { Nannochloropsis, Phaeodactylum, } \\
\text { Nitzschia }\end{array}$ \\
\hline Docosahexanoic acid & $22: 6 \omega 3,6,9,12,15,18$ & Crypthecodinium, Schizochytrium \\
\hline Table 2: Other Microalgal Products & Properties And Uses & Example Microalgal Source \\
\hline Phycoerythrin peptide [26] & $\begin{array}{l}\text { Anti-inflammatory, } \\
\text { antitumor, antiviral }\end{array}$ & $\begin{array}{l}\text { Blue-green algae (Cyanobacteria, } \\
\text { not considered a true algae but } \\
\text { similarly cultivated) }\end{array}$ \\
\hline Astaxanthin pigment [26] & Powerful antioxidant & Haematococcus pluvialis \\
\hline$\beta$-carotene pigment [27] & Antioxidant, pro-vitamin A & Dunaliella salina \\
\hline Fucoxanthin pigment [28] & $\begin{array}{l}\text { Cytotoxicity, antioxidant, } \\
\text { antiproliferative, } \\
\text { proapoptotic }\end{array}$ & Dunaliella salina \\
\hline
\end{tabular}


This presumes that direct consumption, like with Golden Rice, is the only way to partake of microalgal health benefits. Part of the appeal of engineered microalgal food ingredients comes from their incorporation into food products already familiar to the consumer. A 2010 patent assigned to the microalgal biotech company Solazyme lists numerous prototypical recipes for common items such as a miso salad dressing, biscuits, pasta, and whole egg liquids; the use of algal flours, proteins, and oils improved these items' nutritional profiles and bioactive compound content without significant detriment to the consumer experience [29]. Bioactive compounds can also be added to foodstuffs by "natural" means-farmed fish fed a PUFA-enriched diet are a demonstrated vehicle for PUFAs to human consumers, although not necessarily in controlled amounts [24].

Defatting and other processing also ensures that the source organisms, engineered or wild type, do not reach the consumer. Many products are from completely unmodified algal strains, and the genetically modified microalgal source for Solazyme's AlgaWise cooking oil is as removed from the final product as any conventional oil-producing crop [30]. Microalgaeproduced bioactive compounds added to products of conventional, or even "organic" provenance, creates functionalized foods that can curtail the concerns GM products instigate (philosophically- and politically-motivated objections notwithstanding). Isolating the engineered microalgae from the environment in contained growth conditions, and from the consumer by harvest and extraction of compounds, makes algal bioengineering the better biotechnological method of food functionalization.

Wilting the Algal Bloom: But even with Brooks et al.'s patent past its fifth anniversary, and the benefits of microalgae-derived functional foods repeatedly demonstrated, they have a practically non-existent presence in the market. Dietary microalgal cultivation suffers from the same issues that hampers its use in all its other applications. The main challenge continues to be the production of high-value compounds in a manner which is both economical (to maintain the company's viability) and sustainable (to maintain the microbial culture's) [25]. Biofuels have a theoretical environmental incentive by sequestering $\mathrm{CO}_{2}$ greenhouse gas in carbon compounds, and microalgae are especially potent agents by consuming almost twice as much $\mathrm{CO}_{2}$ to produce biomass by weight [31]. The savage irony however is that current algaculture produces more $\mathrm{CO}_{2}$ than it consumes-a bootstrapping problem as mechanical agitation, artificial light sources, fertilizer supplementation, biomass harvesting, and compound extraction still depend on cheaper fossil fuels. Simply put, microalgal cultivation is still agriculture, participating in its complex flux of nutrients and energy and hobbled by the same systematic shortcomings (see Figure $4 \mathrm{~b}$ [32]).

This is less of a concern for bioactive compound production, since the energy the algae consumes is not the intended output (although it does still factor into economic feasibility). Instead bioactive compound makers have to contend with the secondary nature of the secondary metabolites. The natural rate of microalgal lipid production is too slow for producers to get around by simply scaling up operations, or by judiciously selecting their growing systems [33]. Bioactive compound producers are instead turning to genetic engineering like their biofuel colleagues, to make a given volume of culture more productive than the wild type alone [34]. 
Photosynthetic augmentation can improve both microalgal growth rate and pigment compound content, and the intricacies of microalgal fatty acid metabolism present numerous trajectories for scientists to pursue [35]. The less-than-stellar success of fatty acid engineering so far indicates the rudimentary understanding of this Gordian knot, which will be necessary for mass microalgal production of anything of value.

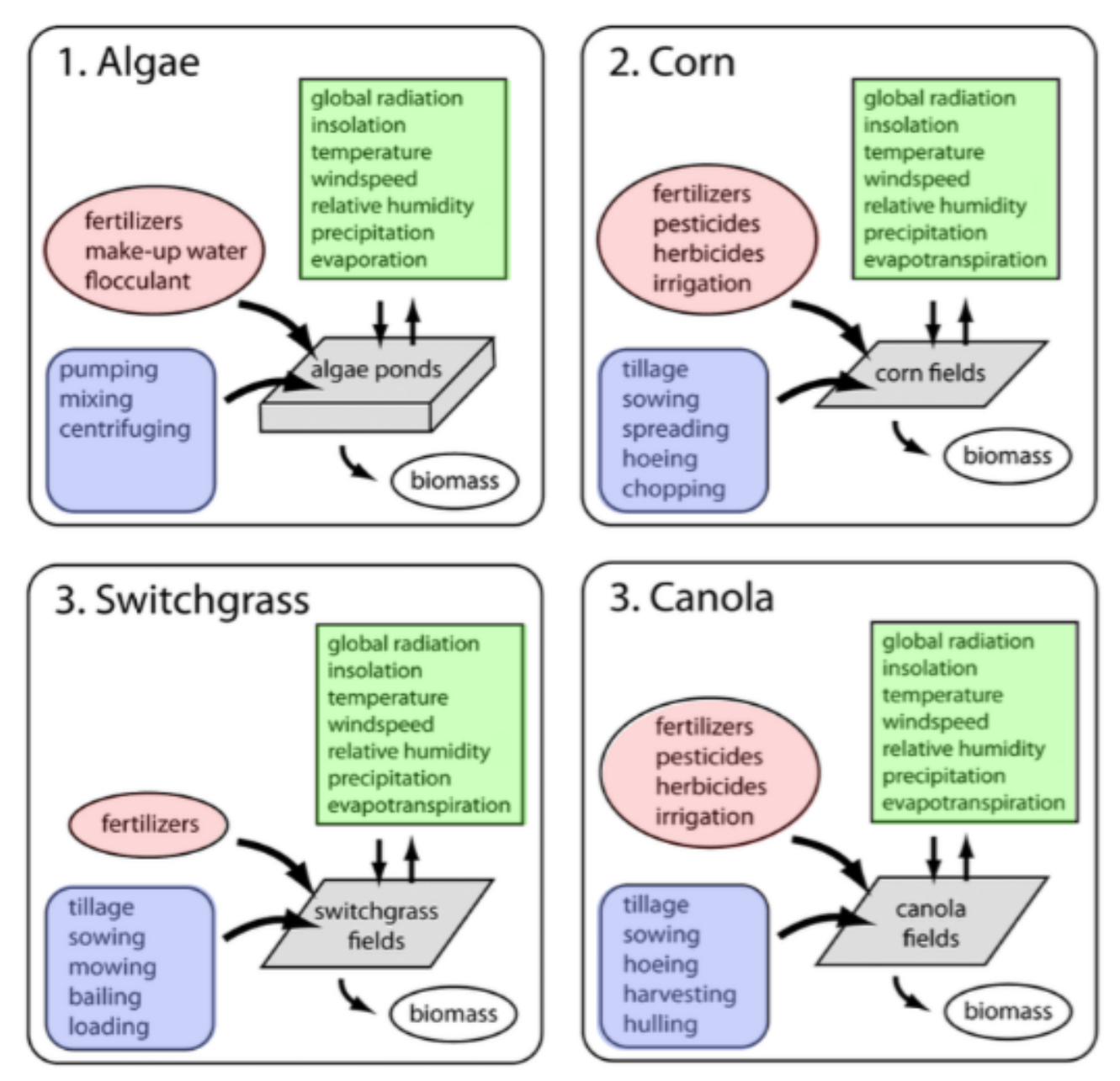

Figure 4b: Processes and materials involved in algaculture, compared and contrasted with other bioenergy crops. Terms in red are physical materials, terms in green are environmental concerns, and terms in blue are mechanical processing steps. Although microalgal cultivation is radically different in handling its biomass, many of the same physical materials are necessary-especially nitrogen and phosphorus fertilizers-and all are subject to the same environmental effects. [30, coloration added by author]

\section{CONCLUSIONS:}

Current functional food application is frequently hampered by a dearth of foods suitable to the purpose. The concurrent advent of biotechnology means that producers and clinicians are not constrained by limited and precarious natural development. Biotechnology has already produced altered dietary staples that can safely induce real health benefits, but the social approval of genetically modified foodstuffs is inconsistent at best.

Modifying microalgae to produce micro and macronutrients, for harvest and incorporation into functional food products, provides the ideal specificity and reliability for bioactive 
compound use. However, its application in biomedical science is impeded by technical difficulty. It remains to be seen if microorganism engineering will be able to meet the needs of its many stakeholders, including the functional food community. Nonetheless, the prospect of a flourishing functional food market, and the healthier population it will bring about, certainly makes it worth a try.

Competing interests: None to declare.

Author contributions: TR proposed this article's topic, performed literature review, and drafted the article content. DM refined the article topic for appropriateness, advised and edited content, and corrected the article formatting for FFHD style compliance.

Financial sponsor: This article was not sponsored, nor was the authors compensated for their work by any entity.

Acknowledgements: We would also like to thank Professor Christina Tzagarakis-Foster at USF for her continued academic and professional guidance.

\section{REFERENCES:}

1. Lawrence S. and Lahteenmaki R. 2014. Public biotech 2013--the numbers. Nature Biotechnology. 32(7):626-32.

2. Martirosyan D.M. and Singh J. 2015. A New Definition of Functional Food by FFC: What Makes a New Definition Unique? Functional Foods in Health and Disease. 5(6):209-23.

3. McConnon A., Cade J. and Pearman A. 2002. Stakeholder interactions and the development of functional foods. Public health nutrition. 5(3):469-77.

4. Wegener C. and Jansen G. 2013. Antioxidants in Different Potato Genotypes: Effect of Drought and Wounding Stress. Agriculture. 3(1):131-46.

5. Kim J., Cha Y.J., Lee K.H. and Park E. 2013. Effect of onion peel extract supplementation on the lipid profile and antioxidative status of healthy young women: a randomized, placebo-controlled, double-blind, crossover trial. Nutrition Research and Practice. 7(5):373.

6. 2011. U.S. Onion Statistics. usda.mannlib.cornell.edu.

7. Bhagwat S.A.,Haytowitz D.B. and Holden J.M. 2014. USDA database for the flavonoid content of selected foods. Release 3.1. 2014.

8. Fares C., Menga V., Martina A., Pellegrini N., Scazzina F. and Torriani S. 2015. Nutritional profile and cooking quality of a new functional pasta naturally enriched in phenolic acids, added with $\beta$-glucan and Bacillus coagulans GBI-30, 6086. Journal of Cereal Science. 65(C):260-6.

9. Svoboda P. and Flemr M. 2010. The role of miRNAs and endogenous siRNAs in maternal-to-zygotic reprogramming and the establishment of pluripotency. EMBO reports. 11(8):590-7.

10. Stein A.J., Sachdev H.P.S. and Qaim M. 2008. Genetic Engineering for the Poor: 
Golden Rice and Public Health in India. World Development. 36(1):144-58.

11. Paine J.A., Shipton C.A., Chaggar S., Howells R.M., Kennedy M.J., Vernon G.,Wright S.Y., Hinchliffe E., Adams J.L., Silverstone A.L. and Drake R. 2005. Improving the nutritional value of Golden Rice through increased pro-vitamin A content. Nature Biotechnology. 23(4):482-7.

12. Meng Z., Meng Z., Zhang R., Liang C., Wan J., Wang Y., Zhai H. and Guo S. 2015. Expression of the Rice Arginase Gene $O S A R G$ in Cotton Influences the Morphology and Nitrogen Transition of Seedlings. PLoS ONE. 10(11):e0141530.

13. Aldemita R.R., Reaño I., Solis R.O. and Hautea R.A. 2015. Trends in global approvals of biotech crops (1992-2014). GM crops \& food. 6(3):150-66.

14. 2011. Approvals of GMOs in the European Union. europabio.org.

15. Blancke S., Van Breusegem F., De Jaeger G., Braeckman J. and Van Montagu M. 2015. Fatal attraction: the intuitive appeal of GMO opposition. Trends in Plant Science. 20(7):414-8.

16. Wesseler J. and Zilberman D. 2014. The economic power of the Golden Rice opposition. Environment and Development Economics. 19(6):724-42.

17. Dubock A. 2014. The politics of Golden Rice. GM Crops \& Food. 5(3):210-22.

18. Lowder L.G., Zhang D., Baltes N.J., Paul J.W., Tang X., Zheng X., Voytas D.F., Hsieh T.F., Zhang Y. and Qi Y. 2015. A CRISPR/Cas9 Toolbox for Multiplexed Plant Genome Editing and Transcriptional Regulation. Plant Physiology. 169(2):971-85.

19. Lozano-Juste J. and Cutler S.R. 2014. Plant genome engineering in full bloom. Trends in Plant Science. 19(5):284-7.

20. Osakabe Y. and Osakabe K. 2015. Genome editing with engineered nucleases in plants. Plant and Cell Physiology. 56(3):389-400.

21. Kanchiswamy C.N., Malnoy M., Velasco R., Kim J.S. and Viola R. 2015. Non-GMO genetically edited crop plants. Trends in Biotechnology. 33(9):489-91.

22. Podevin N., Devos Y., Davies H.V. and Nielsen K.M. 2012. Transgenic or not? No simple answer! New biotechnology-based plant breeding techniques and the regulatory landscape. EMBO reports. 13(12):1057-61.

23. Pauwels K., Podevin N., Breyer D., Carroll D. and Herman P. 2014. Engineering nucleases for gene targeting: safety and regulatory considerations. New Biotechnology. 31(1):18-27.

24. Bellou S., Baeshen M.N., Elazzazy A.M., Aggeli D., Sayegh F. and Aggelis G. 2014. Microalgal lipids biochemistry and biotechnological perspectives. Biotechnology Advances. 32(8):1476-93.

25. Lam M.K. and Lee K.T. 2012. Microalgae biofuels: A critical review of issues, problems and the way forward. Biotechnology Advances. 30(3):673-90.

26. Spolaore P., Joannis-Cassan C., Duran E. and Isambert A. 2006. Commercial applications of microalgae. JBIOSC. 101(2):87-96.

27. Lamers P.P. „Janssen M., De Vos R.C.H., Bino R.J. and Wijffels R.H. 2008. Exploring and exploiting carotenoid accumulation in Dunaliella salina for cell-factory applications. Trends in Biotechnology. 26(11):631-8.

28. Freitas A.C., Rodrigues D., Rocha-Santos T.A.P., Gomes A.M.P. and Duarte A.C. 
2012. Marine biotechnology advances towards applications in new functional foods. Biotechnology Advances. 30(6):1506-15.

29. Brooks G., Franklin S., Avila J. and Decker S.M. 2010. High Protein and High Fiber Algal Food Materials. (12):

30. 2015. Algae oil: The next big healthy cooking oil? foodnavigator-usa.com. http://www.foodnavigator-usa.com/Suppliers2/Solazyme-algae-oil-The-next-bighealthy-cooking-oil.

31. Clarens A.F., Resurreccion E.P., White M.A. and Colosi L.M. 2010. Environmental Life Cycle Comparison of Algae to Other Bioenergy Feedstocks. Environmental Science \& Technology. 44(5):1813-9.

32. Rosenberg J.N., Mathias A., Korth K., Betenbaugh M.J. and Oyler G.A. 2011. Microalgal biomass production and carbon dioxide sequestration from an integrated ethanol biorefinery in Iowa: A technical appraisal and economic feasibility evaluation. Biomass and Bioenergy. 35(9):3865-76.

33. Davis R., Aden A. and Pienkos P.T. 2011. Techno-economic analysis of autotrophic microalgae for fuel production. Applied Energy. 88(10):3524-31.

34. Radakovits R., Jinkerson R.E., Darzins A. and Posewitz M.C. 2010. Genetic engineering of algae for enhanced biofuel production. Eukaryotic Cell. 9(4):486-501.

35. Birkou M., Bokas D. and Aggelis G. 2012. Improving Fatty Acid Composition of Lipids Synthesized by Brachionus plicatilis in Large Scale Experiments. Journal of the American Oil Chemists' Society. 89(11):2047-55. 